\title{
Health behaviours in 131,182 UK women planning pregnancy
}

\author{
Beth McDougall', Kimberley Kavanagh', Judith Stephenson², Lucilla Poston ${ }^{3}$, Angela C. Flynn ${ }^{3,4+}$ and \\ Sara L. White $3^{3^{*}+}$
}

\begin{abstract}
Background: A woman's health at the time of conception lays the foundation for a healthy pregnancy and the lifelong health of her child. We investigated the health behaviours of UK women planning pregnancy.

Methods: We analysed survey data from the 'Planning for Pregnancy' online tool (Tommy's, UK). We described all women planning pregnancy and compared the frequency of non-adherence to preconception recommendations in women who had already stopped contraception (active planners) and those who had not (non-active planners).

Results: One hundred thirty-one thousand one hundred eighty-two women from across the UK were included, of whom $64.8 \%$ were actively planning pregnancy. Of the whole cohort, twenty percent were smokers and less than one third took folic acid supplements (31.5\%). Forty two percent engaged in less than the recommended 150 min of weekly physical activity and only $53.3 \%$ consumed five portions of fruit or vegetables 4 days a week. Smokers were 1.87 times more likely to be active planners than non-smokers ( $95 \% \mathrm{Cl} 1.79-1.94)$, and women who took folic acid were 7 times more likely to be active planners (95\% Cl 6.97-7.59) compared to women who did not. Smoking, drug use and lack of folic acid supplementation were common in younger women and those who were underweight.

Conclusions: This unique survey of UK women has identified poor adherence to preconception recommendations in those planning pregnancies and supports the need for a greater public health focus on preconception health. This study provides a contemporary basis from which to inform preconception health advice and a benchmark to measure changes over time.
\end{abstract}

Keywords: Preconception, Planning for pregnancy, Contraception, Pregnancy

\section{Background}

Health behaviours before pregnancy may profoundly influence pregnancy outcome, and also the future health of mother and child [1], as detailed in a recent Lancet series [2]. Unhealthy and risky behaviours may influence early embryonic viability or development, or if persistent after conception, the health of the fetus, and are difficult

\footnotetext{
${ }^{*}$ Correspondence: sara.white@kcl.ac.uk

${ }^{\dagger}$ AngelaC. Flynn and Sara L. White contributed equally to this work.

${ }^{3}$ Department of Women and Children's Health, School of Life Course

Sciences, King's College London, 10th Floor North Wing, St Thomas'

Hospital, Westminster Bridge Road, SE1 7EH London, UK

Full list of author information is available at the end of the article
}

to modify once pregnancy is established [3, 4]. Public Health England (PHE) recommendations prior to pregnancy include maintaining a healthy diet, participating in frequent physical activity, taking folic acid supplements, encouraging emotional wellbeing, and ensuring cervical screening, sexual health tests and vaccinations are up to date [4]. High risk behaviours in the preconception period include smoking, alcohol consumption and illicit substance use. Obesity is also a risk, being associated with infertility, suboptimal embryonic health and complications in pregnancy [4].

Despite proposals for health policies that increase awareness of the importance of preconception health,

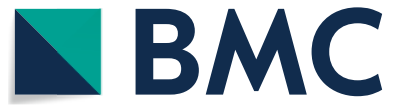

(0) The Author(s) 2021. Open Access This article is licensed under a Creative Commons Attribution 4.0 International License, which permits use, sharing, adaptation, distribution and reproduction in any medium or format, as long as you give appropriate credit to the original author(s) and the source, provide a link to the Creative Commons licence, and indicate if changes were made. The images or other third party material in this article are included in the article's Creative Commons licence, unless indicated otherwise in a credit line to the material. If material is not included in the article's Creative Commons licence and your intended use is not permitted by statutory regulation or exceeds the permitted use, you will need to obtain permission directly from the copyright holder. To view a copy of this licence, visit http://creativecommons.org/licenses/by/4.0/. The Creative Commons Public Domain Dedication waiver (http://creativeco mmons.org/publicdomain/zero/1.0/) applies to the data made available in this article, unless otherwise stated in a credit line to the data. 
there is a lack of knowledge among, and resources available, to women and their health care providers, particularly in primary care [2, 5-7]. A systematic review of healthcare providers' views on preconception care provision suggested inadequacy, with provision of more barriers than facilitators [5]. In the Lancet series, the UK Preconception Partnership proposed a preconception health strategy with targeted public health policies and identification of women planning a pregnancy who would benefit from support, while incorporating the preconception period in existing strategies to counteract obesity and smoking. The Partnership also recommended that the UK adopt an annual report card evaluating metrics from various data sources which holds the government and relative authorities to account for the delivery of preconception advice and interventions [2].

Research has been conducted on the uptake of preconception care $[8,9]$, and studies are beginning to emerge on the effectiveness of intervention programmes [10-13]. In the UK, as in most developed countries, the metrics detailing women's health behaviours prior to conception remain largely conjectural. Most previous studies on preconception health behaviours are retrospective in design, asking pregnant women to look back to their behaviours before conceiving, and therefore being subject to recall bias [3]. Alternatively, inferences of preconception health are drawn from national datasets of women of reproductive age [14], irrespective of pregnancy intention. We aimed to examine the health behaviours and lifestyle factors of a large prospective cohort of UK women planning a pregnancy who completed a digital pregnancy planning health tool. The tool was accessed on the website of a charitable organisation with extensive national and international reach (Tommy's, UK). The study was designed in response to the call for a set of core metrics with which to monitor behavioural changes as a result of public health intervention, and thereby to contribute to the preconception report card as proposed by the Preconception Partnership.

\section{Methods}

This study used data collected from women planning a pregnancy who used an on-line digital health tool (Tommy's 'Planning for Pregnancy' tool; https://www. tommys.org/pregnancy-infor mation/planning-pregn ancy/planning-for-pregnancy-tool) between the 22nd of June 2018 and the 31st of August 2019. The online tool (a questionnaire) is designed to identify women at high risk of pregnancy complications, and to provide tailored advice to improve health and pregnancy outcomes. The questionnaire includes closed-ended questions designed to capture pregnancy planning and health. With support from PHE and the Royal College of Obstetricians and
Gynaecologists (RCOG), it was developed by Tommy's in consultation with multidisciplinary health and medical experts, and with women from the general public. Consumer testing was performed before going live.

\section{Data collection}

The Tommy's tool is openly accessible on the internet. In order to increase awareness and use, social media advertisement via Facebook was used to target women planning pregnancy and the resultant sample of women was self-selected. This included mainstream targeting (females, age 16-45), women from minority ethnic groups, low income women and those with a high BMI. Only data from women living in the UK were included in the analysis. To establish stage of pregnancy planning, women were asked if they had already stopped contraception or when they planned to stop. For the purposes of this study, and to assess preparedness for pregnancy, women who had already stopped contraception were defined as 'active planners' and those who had not stopped as 'non-active planners'. 'Active planners' were further divided into those who had stopped contraception less than or more than a year ago.

Data collected on health behaviours included smoking (current, yes/no), alcohol and caffeine consumption, illicit or recreational drug use, supplementation with folic acid (yes/no), consumption of 5 portions of fruit and vegetables at least 4 days a week (yes/no/don't know) and weekly physical activity levels (less than $150 \mathrm{~min}, 150 \mathrm{~min}$ (moderate), 150 min (vigorous)). Data on physical health were also collected.

Demographic data included age in years (18-24, 25-34, $35-40,41+$ ), self-reported weight and height and BMI (calculated; weight/height, $\mathrm{kg} / \mathrm{m}^{2}$ ), classified according to the World Health Organization definitions: underweight $\left(<18.5 \mathrm{~kg} / \mathrm{m}^{2}\right)$; normal weight $\left(18.5-24.9 \mathrm{~kg} / \mathrm{m}^{2}\right)$ (referred to as recommended BMI); overweight (25-29.9 kg/ $\left.\mathrm{m}^{2}\right)$; and obese $\left(\geq 30 \mathrm{~kg} / \mathrm{m}^{2}\right)$ [15]. Geographical location (country and city) was determined using anonymised IP address.

\section{Outcome measures}

Outcome variables for the logistic regression were pregnancy intention (active planners or non-active planners) or length of time trying to conceive (active planners who had stopped contraception less than or more than a year ago).

\section{Data and statistical analysis}

Duplicate entries were removed as identified by identical time, date, and anonymised IP address. Any biologically implausible BMI values were excluded (4.5\% of respondents; height outside range $140-190 \mathrm{~cm}$ and weight 
outside range 30-190 kg) based on international weight and height data and a cohort of obese pregnant women [16-18]. The respondent's location was determined from anonymised IP addresses which also enabled identification of non-UK resident women, who were excluded. As identified in the Tables, not all questions were answered by all women. The maximal number available for analysis is used for each question.

Descriptive statistics were calculated, with frequencies and percentages presented for categorical variables. Logistic regression was used to assess the association between each of the pregnancy planning outcome measures and health behaviours. Both adjusted and unadjusted odds ratios were calculated for each of the pregnancy planning measures. A Bonferroni correction was applied to adjust the p-values in the univariate logistic regression models to account for multiple testing and identify which variables were significant before proceeding to adjusted models. The multivariable regression models were adjusted for age, BMI, smoking, drug use, alcohol consumption, caffeine consumption, physical activity level, consumption of fruit and vegetables and folic acid supplementation. Health behaviours were also compared between those active planners who had stopped contraception more, or less than one year ago. Interaction effects between age and BMI and each of the health behaviours; smoking, drug use, alcohol consumption and folic acid supplementation were also examined. Statistical analysis was performed using RStudio.

\section{Results}

\section{Study population}

Between 22nd of June 2018 and the 31st of August 2019, 214,228 entries were recorded in the Tommy's tool database (Fig. 1). After deduplication of entries based on time, date and IP address this reduced to 164,352 users, of whom 131,182 were in the UK, and geographically distributed across England, Wales, Scotland and Northern Ireland (Fig. 2). Of those with a known age $(n=127,102), 38 \%$ of women were aged $18-24$ years, $51 \%$ were aged $25-34$ years, $10 \%$ were aged 35-40 years and the remaining $1 \%$ were aged 41 years or above. For the women with height and weight data 5,874 were excluded due to implausible values, giving $n=111,032$. Of this, $3 \%$ were underweight, $42 \%$ were of recommended BMI, $26 \%$ were overweight and $29 \%$ obese. Sixty-four point eight percent ( $n=85,040$ of 131,151 who responded) of women

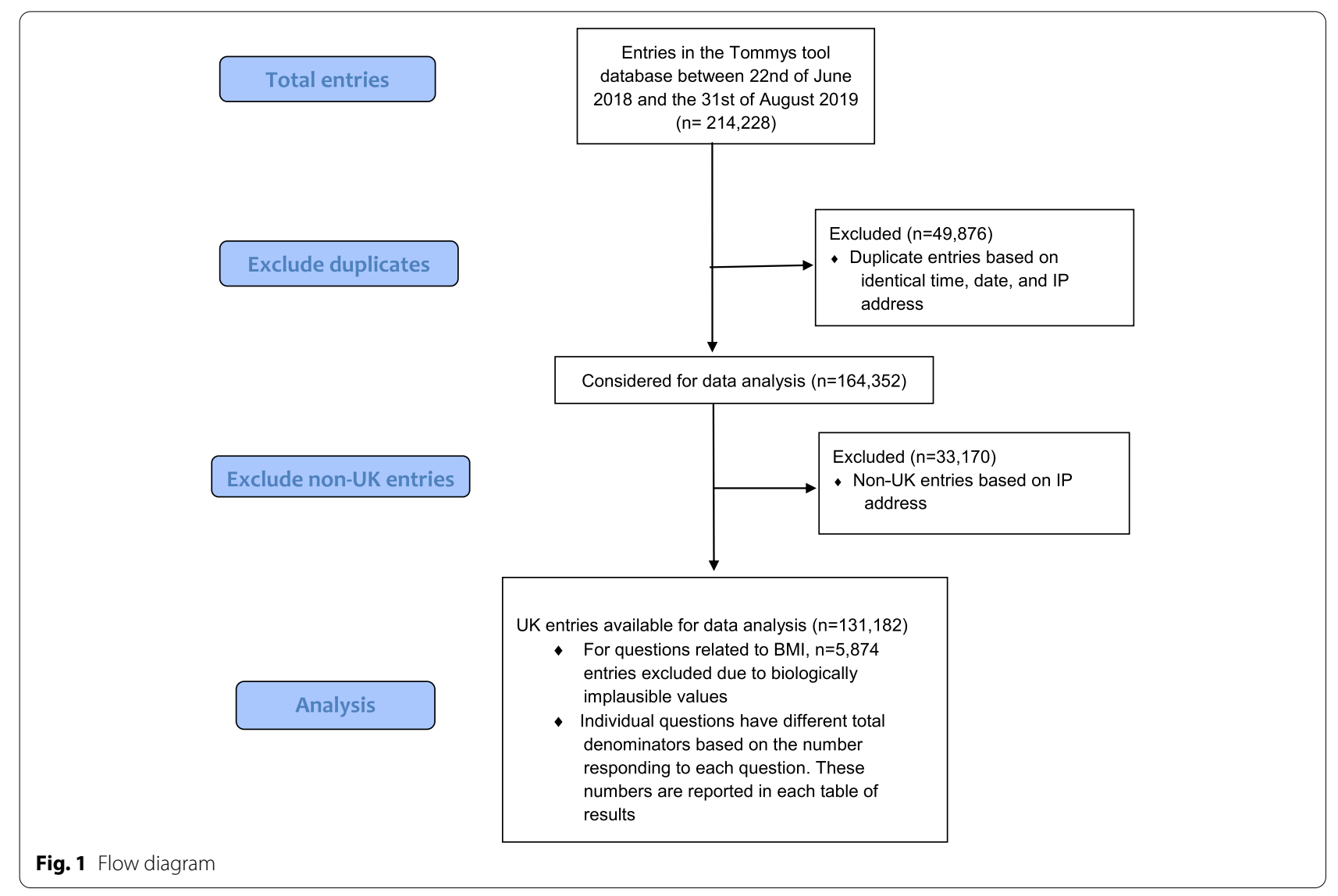




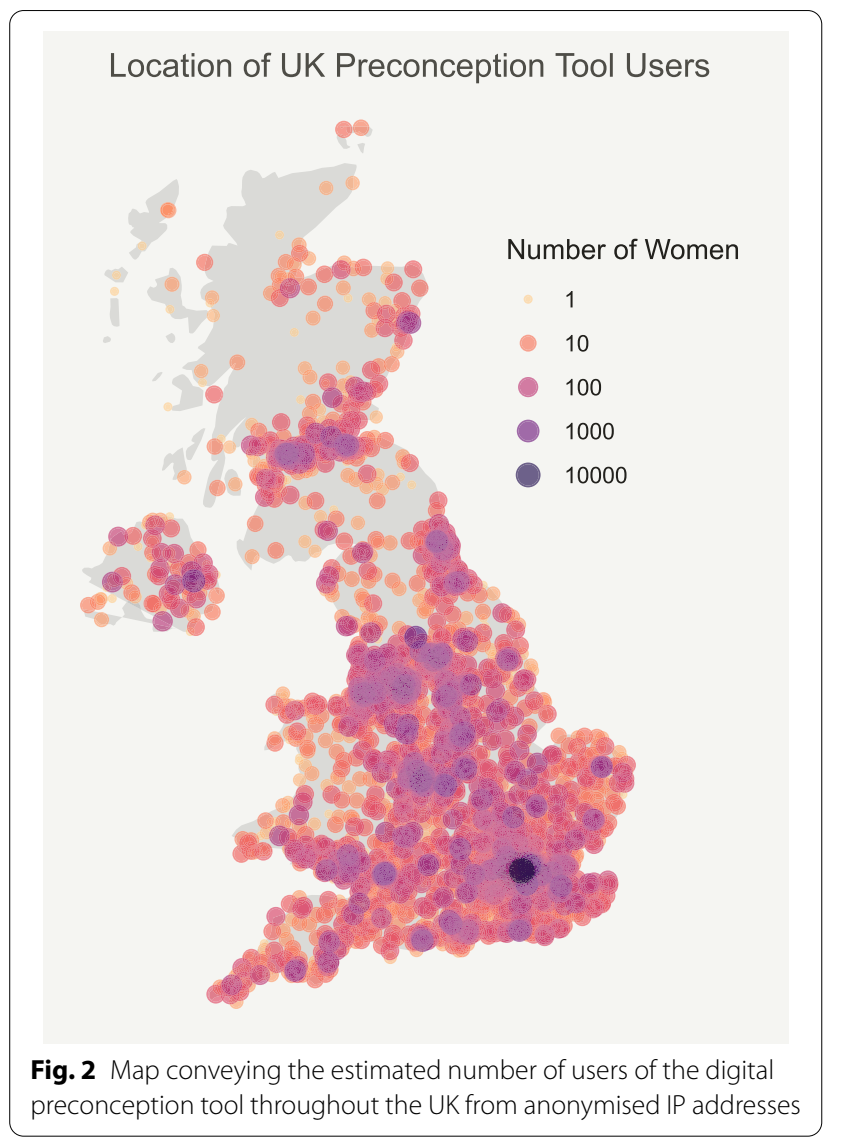

were actively planning for pregnancy while $35.2 \%$ $(n=46,111)$ were not actively planning. Of those who were active planners, $46.9 \%(n=39,912)$ had stopped contraception over a year ago.

\section{Health behaviours and physical health}

Twenty percent $(n=25,445)$ of women planning a pregnancy reported smoking and 3.7\% $(n=4,698)$ reported illicit/recreational drug use. Alcohol consumption was reported by $54.5 \% \quad(n=69,195)$, while caffeine consumption was reported by $79.6 \%$ $(n=100,901) .42 \%$ of women $(n=49,072)$ participated in less than the recommended 150 min of weekly activity and just over half of women $(53.3 \%, n=61,747)$ reported consuming five portions of fruit and vegetables at least 4 days each week. Folic acid supplement use was low $(31.5 \%, n=31,329) .14 .9 \%(n=17,166)$ of women reported having a physical health condition or a condition in a previous pregnancy, of whom less than half $(n=6942,40.6 \%)$ had spoken to a doctor or specialist about their plans for pregnancy (Table 1).

\section{Women actively planning a pregnancy (not using} contraception)

Women who took folic acid supplements were more likely to be active planners than those who did not take supplements (adjusted OR 7.27, 95\% CI 6.97-7.59) with $44 \%$ of active planners taking supplements compared to $9.2 \%$ of non-active planners (Table 2). Folic acid supplementation was lower in active planners aged $18-24$ years, of whom $29.1 \%$ (95\% CI $28.9-29.3$ ) reported taking supplements, almost half the percentage compared to older groups (Table 3). Folic acid supplementation was also lower in active planners who were underweight and obese where $36.9 \%$ (95\% CI $36.2-37.6)$ and $39.3 \%(39.1-39.7)$ respectively reported taking supplements (Table 4).

Smokers were more likely to be active planners compared to non-smokers (adjusted OR 1.87, 95\% CI 1.791.94) with $21.7 \%$ (95\% CI 21.4-22.0) of active planners reporting smoking (Table 2). The youngest group of active planners were the most likely to smoke; $31 \%$ compared to $15-18 \%$ in the older age groups (Table 3 ). There was also a high frequency of smoking amongst active planners who were underweight, 31.3\% (95\% CI 30.7-32.0) (Table 4).

Women who consumed alcohol were less likely to be active planners (adjusted OR 0.58, 95\% CI 0.56-0.60). Illicit or recreational drug use was most common in the active planners in the 18-24 age group and in those who were underweight, with a similar frequency in both groups $(\sim 5 \%)$. Albeit statistically significant, differences in other health behaviours were relatively small between active and non-active planners.

Active planners were twice as likely to have stopped contraception over a year ago if they were aged between 35-40 years (adjusted OR 2.17, 95\% CI 2.052.30 ) and almost 3 times as likely if they were aged 41 and above (adjusted OR 2.88, 95\% CI 2.52-3.29), compared to those in the 18-24 age group. Women in the underweight (adjusted OR 1.19, 95\% CI 1.09-1.29) and obese (adjusted OR 1.30, 95\% CI 1.24-1.36) BMI groups were also more likely to have stopped contraception over a year ago than those in the recommended BMI group. Active planners who smoked and used illicit/recreational drugs were more likely to have stopped contraception over a year ago (adjusted OR $1.25,95 \%$ CI $1.20-1.31$ and adjusted OR 1.17, 95\% CI $1.07-1.28$, respectively). In this group of active planners, drinking alcohol and taking folic acid was less likely in those who had stopped contraception over a year ago (adjusted OR 0.74, 95\% CI $0.72-0.77$ and adjusted OR $0.88,95 \%$ CI $0.85-0.91$, respectively) (Table 5). 
Table 1 Participant characteristics, health behaviours and numbers of women who provided a response

\begin{tabular}{|c|c|c|}
\hline & Number of women & $\begin{array}{l}\text { Percentage of } \\
\text { women }\end{array}$ \\
\hline \multicolumn{3}{|l|}{ Age } \\
\hline $18-24$ & 47,977 & $37.8 \%$ \\
\hline $25-34$ & 64,684 & $50.9 \%$ \\
\hline $35-40$ & 12,905 & $10.2 \%$ \\
\hline $41+$ & 1,536 & $1.2 \%$ \\
\hline Question not answered & 4,080 & - \\
\hline \multicolumn{3}{|l|}{ BMI } \\
\hline$<18.5$ & 3,826 & $3.5 \%$ \\
\hline $18.5-24.9$ & 46,739 & $42.1 \%$ \\
\hline $25-29$ & 28,559 & $25.7 \%$ \\
\hline $30+$ & 31,908 & $28.7 \%$ \\
\hline BMl excluded & 5,874 & - \\
\hline Question not answered & 14,276 & - \\
\hline \multicolumn{3}{|l|}{ Smoking } \\
\hline No & 101,611 & $80.0 \%$ \\
\hline Yes & 25,445 & $20.0 \%$ \\
\hline Question not answered & 4,126 & - \\
\hline \multicolumn{3}{|l|}{ Illicit or recreational drug use } \\
\hline No & 122,288 & $96.3 \%$ \\
\hline Yes & 4,698 & $3.7 \%$ \\
\hline Question not answered & 4,196 & - \\
\hline \multicolumn{3}{|l|}{ Alcohol consumption } \\
\hline No & 57,772 & $45.5 \%$ \\
\hline Yes & 69,195 & $54.5 \%$ \\
\hline Question not answered & 4,215 & - \\
\hline \multicolumn{3}{|l|}{ Caffeine consumption } \\
\hline No & 25,921 & $20.4 \%$ \\
\hline Yes & 100,901 & $79.6 \%$ \\
\hline Question not answered & 4,360 & - \\
\hline \multicolumn{3}{|l|}{ Weekly level of activity } \\
\hline Less than 150 min of activity & 49,072 & $42.0 \%$ \\
\hline At least 150 min of moderate activity & 50,813 & $43.5 \%$ \\
\hline 150 or more minutes of vigorous activity & 16,948 & $14.5 \%$ \\
\hline Question not answered & 14,349 & - \\
\hline \multicolumn{3}{|c|}{ Daily consumption of five portions of fruit and vegetables } \\
\hline No & 36,590 & $31.6 \%$ \\
\hline Yes & 61,747 & $53.3 \%$ \\
\hline Don't know & 17,463 & $15.1 \%$ \\
\hline Question not answered & 15,388 & - \\
\hline \multicolumn{3}{|l|}{ Folic acid supplementation } \\
\hline No & 68,033 & $68.5 \%$ \\
\hline Yes & 31,329 & $31.5 \%$ \\
\hline Question not answered & 31,820 & - \\
\hline \multicolumn{3}{|l|}{ Physical health condition ${ }^{a}$} \\
\hline No & 98,316 & $85.1 \%$ \\
\hline Yes & 17,166 & $14.9 \%$ \\
\hline Question not answered & 15,700 & \\
\hline \multicolumn{3}{|c|}{ Has a Physical health condition and consulted doctor or specialist about pregnancy plans } \\
\hline No & 10,151 & $59.4 \%$ \\
\hline Yes & 6,942 & $40.6 \%$ \\
\hline Question not answered & 114,089 & - \\
\hline
\end{tabular}

${ }^{a}$ This could include asthma, high blood pressure, heart problems, auto-immune conditions, acne, pre-eclampsia, gestational diabetes or epilepsy 
Table 2 Participant health behaviours stratified by pregnancy intention

\begin{tabular}{|c|c|c|c|c|c|}
\hline & \multirow[b]{2}{*}{ Number of women } & \multicolumn{2}{|c|}{ Percentage of women $(95 \% \mathrm{Cl})$} & \multirow[b]{2}{*}{$\begin{array}{l}\text { Unadjusted OR } \\
(95 \% \mathrm{Cl})\end{array}$} & \multirow[b]{2}{*}{$\begin{array}{l}\text { Adjusted OR } \\
(95 \% \mathrm{Cl})^{\mathrm{a}}\end{array}$} \\
\hline & & $\begin{array}{l}\text { Non-active planners } \\
(n=46,111)\end{array}$ & $\begin{array}{l}\text { Active planners } \\
(n=85,040)\end{array}$ & & \\
\hline \multicolumn{6}{|l|}{ Age $(n=127,101)$} \\
\hline $18-24$ & 47,977 & $50.4 \%(49.9-50.8)$ & $30.7 \%(30.4-31.0)$ & 1.00 (ref) & 1.00 (ref) \\
\hline $25-34$ & 64,683 & $43.8 \%(43.3-44.2)$ & $54.8 \%(54.5-55.2)$ & $2.06(2.01-2.11)$ & $1.82(1.76-1.88)$ \\
\hline $35-40$ & 12,905 & $5.3 \%(5.1-5.6)$ & $12.8 \%(12.6-13.1)$ & $3.94(3.76-4.13)$ & $3.40(3.19-3.62)$ \\
\hline $41+$ & 1536 & $0.5 \%(0.4-0.6)$ & $1.6 \%(1.5-1.7)$ & $5.29(4.59-6.10)$ & $4.60(3.84-5.50)$ \\
\hline Question not answered & 4081 & - & - & - & - \\
\hline \multicolumn{6}{|l|}{ BMI $(n=111,031)$} \\
\hline 18.5-24.9 (Recommended) & 46,739 & $45.6 \%(45.1-46.1)$ & $40.2 \%(39.8-40.5)$ & 1.00 (ref) & 1.00 (ref) \\
\hline$<18.5$ (Underweight) & 3826 & $3.8 \%(3.6-4.0)$ & $3.2 \%(3.1-3.4)$ & $0.96(0.90-1.02)$ & $1.05(0.97-1.13)$ \\
\hline 25-29 (Overweight) & 28,559 & $25.5 \%(25.1-25.9)$ & $25.8 \%(25.5-26.2)$ & $1.15(1.12-1.19)$ & $1.12(1.08-1.16)$ \\
\hline $30+$ (Obese) & 31,907 & $25.1 \%(24.7-25.5)$ & $30.8 \%(30.4-31.1)$ & $1.39(1.35-1.44)$ & $1.48(1.42-1.55)$ \\
\hline Question not answered/BMl excluded & 20,151 & - & - & - & - \\
\hline \multicolumn{6}{|l|}{ Smoking $(n=127,056)$} \\
\hline No & 101,611 & $83.0 \%(82.7-83.3)$ & $78.3 \%(78.0-78.6)$ & 1.00 (ref) & 1.00 (ref) \\
\hline Yes & 25,445 & $17.0 \%(16.7-17.3)$ & $21.7 \%(21.4-22.0)$ & $1.35(1.31-1.39)$ & $1.87(1.79-1.94)$ \\
\hline Question not answered & 4126 & - & - & - & - \\
\hline \multicolumn{6}{|c|}{ Illicit or recreational drug use $(n=126,985)$} \\
\hline No & 122,287 & $96.0 \%(95.8-96.1)$ & $96.5 \%(96.4-96.6)$ & 1.00 (ref) & 1.00 (ref) \\
\hline Yes & 4698 & $4.0 \%(3.9-4.2)$ & $3.5 \%(3.4-3.6)$ & $0.86(0.81-0.92)$ & $0.99(0.92-1.08)$ \\
\hline Question not answered & 4166 & - & - & - & - \\
\hline \multicolumn{6}{|l|}{ Alcohol consumption $(n=126,966)$} \\
\hline No & 57,771 & $35.8 \%(35.3-36.2)$ & $50.9 \%(50.5-51.2)$ & 1.00 (ref) & 1.00 (ref) \\
\hline Yes & 69,195 & $64.2 \%(63.8-64.7)$ & $49.1 \%(48.8-49.5)$ & $0.54(0.53-0.55)$ & $0.58(0.56-0.60)$ \\
\hline Question not answered & 4185 & - & - & - & - \\
\hline \multicolumn{6}{|l|}{ Caffeine consumption ( $n=126,820)$} \\
\hline No & 25,919 & $19.1 \%(18.7-19.5)$ & $21.2 \%(20.9-21.5)$ & 1.00 (ref) & 1.00 (ref) \\
\hline Yes & 100,901 & $80.9 \%(80.5-81.3)$ & $78.8 \%(78.5-79.1)$ & $0.88(0.85-0.91)$ & $1.00(0.96-1.04)$ \\
\hline Question not answered & 4331 & - & - & - & - \\
\hline \multicolumn{6}{|l|}{ Weekly level of activity $(n=116,832)$} \\
\hline Less than 150 min of activity & 49,071 & $40.9 \%(40.4-41.4)$ & $42.6 \%(42.3-43.0)$ & 1.00 (ref) & 1.00 (ref) \\
\hline At least 150 min of moderate activity & 50,813 & $44.4 \%(43.9-44.9)$ & $43.0 \%(42.6-43.4)$ & $0.93(0.91-0.95)$ & $0.91(0.88-0.94)$ \\
\hline 150 or more minutes of vigorous activity & 16,948 & $14.7 \%(14.4-15.1)$ & $14.4 \%(14.1-14.6)$ & $0.94(0.90-0.97)$ & $0.94(0.90-0.98)$ \\
\hline Question not answered & 14,319 & - & - & - & - \\
\hline \multicolumn{6}{|c|}{ Daily consumption of five portions of fruit and vegetables $(n=115,793)$} \\
\hline No & 36,589 & $32.5 \%(32.0-32.9)$ & $31.1 \%(30.8-31.4)$ & 1.00 (ref) & 1.00 (ref) \\
\hline Yes & 61,747 & $52.0 \%(51.5-52.5)$ & $54.1 \%(53.7-54.4)$ & $1.09(1.06-1.12)$ & $0.92(0.89-0.95)$ \\
\hline Don't know & 17,463 & $15.5 \%(15.2-15.9)$ & $14.8 \%(14.6-15.1)$ & $1.00(0.96-1.04)$ & $1.05(1.00-1.10)$ \\
\hline Question not answered & 15,352 & - & - & - & - \\
\hline \multicolumn{6}{|l|}{ Folic acid supplementation $(n=99,362)$} \\
\hline No & 68,033 & $90.8 \%(90.5-91.1)$ & $56.0 \%(55.6-56.3)$ & 1.00 (ref) & 1.00 (ref) \\
\hline Yes & 31,329 & $9.2 \%(8.9-9.5)$ & $44.0 \%(43.7-44.4)$ & $7.75(7.45-8.06)$ & $7.27(6.97-7.59)$ \\
\hline Question not answered & 31,789 & - & - & - & - \\
\hline
\end{tabular}

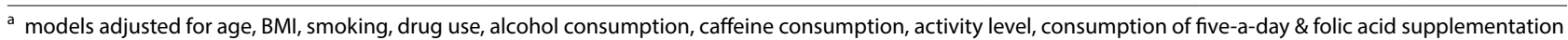


Table 3 Health behaviours of women actively planning pregnancy (not using contraception) stratified by age

\begin{tabular}{|c|c|c|c|c|c|}
\hline & \multirow[b]{3}{*}{$\begin{array}{l}\text { Number } \\
\text { of active } \\
\text { planners }\end{array}$} & \multicolumn{4}{|c|}{ Percentage of active planners $(95 \% \mathrm{Cl})$} \\
\hline & & \multicolumn{4}{|l|}{ Age } \\
\hline & & $18-24(n=25,086)$ & $25-34(n=44,795)$ & $35-40(n=10,479)$ & $41+(n=1310)$ \\
\hline \multicolumn{6}{|l|}{ Smoking $(n=81,118)$} \\
\hline No & 63,561 & $69.1 \%(68.5-69.6)$ & $82.2 \%(82.1-82.3)$ & $83.2 \%(82.9-83.4)$ & $85.0 \%(84.3-85.7)$ \\
\hline Yes & 17,557 & $30.9 \%(30.4-31.5)$ & $17.8 \%(17.7-17.9)$ & $16.9 \%(16.6-17.1)$ & $15.0 \%(14.3-15.7)$ \\
\hline Question not answered & 552 & - & - & - & - \\
\hline \multicolumn{6}{|c|}{ Illicit or recreational drug use $(n=81,145)$} \\
\hline No & 78,301 & $94.7 \%(94.6-94.8)$ & $97.3 \%(97.2-97.3)$ & $97.4 \%(97.3-97.5)$ & $96.8 \%(96.5-97.1)$ \\
\hline Yes & 2844 & $5.3 \%(5.2-5.4)$ & $2.7 \%(2.7-2.8)$ & $2.6 \%(2.5-2.7)$ & $3.2 \%(2.9-3.5)$ \\
\hline Question not answered & 525 & - & - & - & - \\
\hline \multicolumn{6}{|l|}{ Alcohol consumption $(n=81,128)$} \\
\hline No & 41,252 & $57.0 \%(56.8-57.2)$ & $47.5 \%(47.4-47.7)$ & $49.4 \%(49.1-49.7)$ & $57.1 \%(56.2-58.0)$ \\
\hline Yes & 39,876 & $43.0 \%(42.8-43.2)$ & $52.5 \%(52.3-52.6)$ & $50.6 \%(50.3-50.9)$ & $42.9 \%(42.0-43.8)$ \\
\hline Question not answered & 542 & - & - & - & - \\
\hline \multicolumn{6}{|l|}{ Caffeine consumption ( $n=81,029)$} \\
\hline No & 17,154 & $23.4 \%(23.3-23.6)$ & $19.8 \%(19.7-19.9)$ & $21.1 \%(20.8-21.4)$ & $25.7 \%(24.9-26.5)$ \\
\hline Yes & 63,875 & $76.6 \%(76.4-76.8)$ & $80.2 \%(80.1-80.3)$ & $78.9 \%(78.6-79.2)$ & $74.3 \%(73.5-75.1)$ \\
\hline Question not answered & 641 & - & - & - & - \\
\hline \multicolumn{6}{|l|}{ Weekly level of activity $(n=74,600)$} \\
\hline Less than 150 min of activity & 31,783 & $42.4 \%(42.2-42.7)$ & $43.0 \%(42.8-43.2)$ & $41.9 \%(41.5-42.2)$ & $38.0 \%(37.0-38.9)$ \\
\hline At least 150 min of moderate activity & 32,097 & $41.8 \%(41.6-42.1)$ & $43.6 \%(43.4-43.8)$ & $43.1 \%(42.8-43.5)$ & $44.3 \%(43.3-45.2)$ \\
\hline 150 or more minutes of vigorous activity & 10,720 & $15.7 \%(15.6-15.9)$ & $13.4 \%(13.3-13.5)$ & $15.0 \%(14.8-15.2)$ & $17.8 \%(17.1-18.5)$ \\
\hline Question not answered & 7070 & - & - & - & - \\
\hline \multicolumn{6}{|c|}{ Daily consumption of five portions of fruit and vegetables $(n=74,050)$} \\
\hline No & 23,031 & $36.0 \%(35.7-36.2)$ & $30.2 \%(30.0-30.3)$ & $25.0 \%(24.7-25.3)$ & $22.6 \%(21.8-23.4)$ \\
\hline Yes & 40,032 & $43.7 \%(43.4-43.9)$ & $56.7 \%(56.5-56.9)$ & $64.6 \%(64.3-64.9)$ & $69.2 \%(68.3-70.0)$ \\
\hline Don't know & 10,987 & $20.4 \%(20.2-20.6)$ & $13.1 \%(13.0-13.2)$ & $10.4 \%(10.2-10.6)$ & $8.2 \%(7.7-8.8)$ \\
\hline Question not answered & 7620 & - & - & - & - \\
\hline \multicolumn{6}{|l|}{ Folic acid supplementation $(n=63,160)$} \\
\hline No & 35,333 & $70.8 \%(70.7-71.1)$ & $50.9 \%(50.7-51.1)$ & $44.5 \%(44.1-44.8)$ & $45.5 \%(44.5-46.5)$ \\
\hline Yes & 27,827 & $29.1 \%(28.9-29.3)$ & $49.1 \%(48.9-49.3)$ & $55.5 \%(55.2-55.9)$ & $54.5 \%(53.5-55.5)$ \\
\hline Question not answered & 18,510 & - & - & - & - \\
\hline
\end{tabular}

\section{Discussion}

\section{Main findings and interpretation}

We have examined the health behaviours and lifestyle factors of the largest cohort to date of UK women planning a pregnancy. Given that there are approximately 650,000 births in England and Wales every year [19], and the wide geographical distribution of the respondents, this survey is likely to reflect the behaviours of a large proportion of UK women planning pregnancy. Our analysis indicates that many health behaviours were sub-optimal including smoking, lack of folic acid supplementation, alcohol consumption, low levels of physical activity and of fruit and vegetable intake in the diet. These unhealthy behaviours were most evident in younger women and in those who were underweight. A shift towards healthier behaviours was observed in women actively planning pregnancy, except for smoking, however this improvement appears transient, as evidenced by poorer health behaviours in women trying to conceive for more than a year. Together, these data show that UK women, over half of whom plan pregnancy (54\%) [20], are not adequately prepared, which has broad implications for the health of this and future generations.

We found that approximately one in five women reported smoking, a similar proportion to a retrospective UK study in 2014 of 1173 women which found that $21 \%$ of women smoked [7] and in alignment with national data from 2019 which reported $21.2 \%$ in 
Table 4 health behaviours of women actively planning pregnancy stratified by BMI

\begin{tabular}{|c|c|c|c|c|c|}
\hline & \multirow[b]{3}{*}{$\begin{array}{l}\text { Number } \\
\text { of active } \\
\text { planners }\end{array}$} & \multicolumn{4}{|c|}{ Percentage of active planners $(95 \% \mathrm{Cl})$} \\
\hline & & \multicolumn{4}{|l|}{ BMI } \\
\hline & & $<18.5(n=2311)$ & $18.5-24.9(n=28,710)$ & $25-29(n=18,473)$ & $30+(n=21,990)$ \\
\hline \multicolumn{6}{|l|}{ Smoking $(n=71,116)$} \\
\hline No & 56,301 & $68.7 \%(68.0-69.3)$ & $81.4 \%(81.3-81.6)$ & $80.2 \%(80.0-80.4)$ & $76.5 \%(76.3-76.7)$ \\
\hline Yes & 14,815 & $31.3 \%(30.7-32.0)$ & $18.6 \%(18.4-18.7)$ & $19.8 \%(19.6-20.0)$ & $23.5 \%(23.3-23.7)$ \\
\hline Question not answered & 368 & - & - & - & - \\
\hline \multicolumn{6}{|c|}{ Illicit or recreational drug use $(n=71,328)$} \\
\hline No & 68,934 & $94.6 \%(94.3-94.9)$ & $96.2 \%(96.1-96.3)$ & $97.1 \%(97.0-97.2)$ & $97.0 \%(96.9-97.1)$ \\
\hline Yes & 2394 & $5.4 \%(5.1-5.7)$ & $3.8 \%(3.7-3.9)$ & $2.9 \%(2.8-3.0)$ & $3.0 \%(2.9-3.1)$ \\
\hline Question not answered & 156 & - & - & - & - \\
\hline \multicolumn{6}{|l|}{ Alcohol consumption $(n=71,381)$} \\
\hline No & 35,428 & $61.0 \%(60.3-61.7)$ & $46.0 \%(45.8-46.2)$ & $47.5 \%(47.2-47.7)$ & $55.0 \%(54.7-55.2)$ \\
\hline Yes & 35,953 & $39.0 \%(38.3-39.7)$ & $54.0 \%(53.8-54.2)$ & $52.5 \%(52.3-52.8)$ & $45.0 \%(44.8-45.3)$ \\
\hline Question not answered & 103 & - & - & - & - \\
\hline \multicolumn{6}{|l|}{ Caffeine consumption ( $n=71,393)$} \\
\hline No & 14,621 & $22.8 \%(22.2-23.4)$ & $20.1 \%(19.9-20.2)$ & $19.9 \%(19.7-20.1)$ & $21.3 \%(21.1-21.4)$ \\
\hline Yes & 56,772 & $77.2 \%(76.6-77.8)$ & $79.9 \%(79.8-80.1)$ & $80.1 \%(79.9-80.3)$ & $78.7 \%(78.6-78.9)$ \\
\hline Question not answered & 91 & - & - & - & - \\
\hline \multicolumn{6}{|l|}{ Weekly level of activity $(n=71,224)$} \\
\hline Less than 150 min of activity & 30,261 & $40.9 \%(40.2-41.6)$ & $38.4 \%(38.2-38.6)$ & $42.7 \%(42.4-42.9)$ & $47.8 \%(47.6-48.0)$ \\
\hline At least 150 min of moderate activity & 31,041 & $43.5 \%(42.8-44.2)$ & $45.8 \%(45.6-46.0)$ & $43.9 \%(43.7-44.2)$ & $40.4 \%(40.2-40.7)$ \\
\hline 150 or more minutes of vigorous activity & 9922 & $15.6 \%(15.1-16.1)$ & $15.8 \%(15.7-16.0)$ & $13.4 \%(13.2-13.6)$ & $11.7 \%(11.6-11.9)$ \\
\hline Question not answered & 260 & - & - & - & - \\
\hline \multicolumn{6}{|c|}{ Daily consumption of five portions of fruit and vegetables $(n=70,602)$} \\
\hline No & 21,864 & $36.4 \%(35.7-37.1)$ & $30.1 \%(29.9-30.3)$ & $30.5 \%(30.3-30.7)$ & $31.9 \%(31.7-32.1)$ \\
\hline Yes & 38,307 & $46.8 \%(46.1-47.5)$ & $55.4 \%(55.2-55.6)$ & $54.9 \%(54.7-55.2)$ & $53.0 \%(52.8-53.2)$ \\
\hline Don't know & 10,431 & $16.8 \%(16.3-17.4)$ & $14.5 \%(14.3-14.6)$ & $14.6 \%(14.4-14.8)$ & $15.1 \%(15.0-15.3)$ \\
\hline Question not answered & 882 & - & - & - & - \\
\hline \multicolumn{6}{|l|}{ Folic acid supplementation $(n=60,318)$} \\
\hline No & 33,487 & $63.1 \%(62.4-63.8)$ & $53.3 \%(53.1-53.5)$ & $54.7 \%(54.4-54.9)$ & $60.6 \%(60.3-60.9)$ \\
\hline Yes & 26,831 & $36.9 \%(36.2-37.6)$ & $46.7 \%(46.5-46.9)$ & $45.3 \%(45.1-45.6)$ & $39.3 \%(39.1-39.7)$ \\
\hline Question not answered & 11,166 & - & - & - & - \\
\hline
\end{tabular}

women aged 25-34 years [21]. Our finding that smokers were more likely to be actively trying to conceive than non-smokers was unexpected and contrasts with a recent report (2020) of 294 women in Australia where no difference was found between these two groups [3]. The observation that smoking was most prevalent in those who stopped contraception greater than one year might reflect lack of sustained change in behaviour after a year of failing to conceive. It alternatively reflects more difficulty in conceiving in those with poorer health behaviours.

Despite widespread evidence-based public health guidance on the benefits of taking folic acid in the preconception period, only one in three women planning pregnancy reported supplement use. Women taking supplements were more likely to be active planners, although more than half of this group i.e. women who had stopped contraception, took no folic acid. Clearly, the recommendation that all women planning pregnancy should take folic acid remains unheeded by many. Little has changed in the past two decades; in a UK survey undertaken between 1998 and 2002, 44\% of women who became pregnant within three months of participating had taken folic acid supplements [22]. This sustained lack of adherence to public health advice amongst those planning pregnancy, notwithstanding unintended pregnancies, strongly supports mandatory folic acid fortification in the UK. Estimates suggest that 
Table 5 Participant health behaviours stratified by duration of pregnancy planning

\begin{tabular}{|c|c|c|c|c|c|}
\hline & \multirow[b]{2}{*}{$\begin{array}{l}\text { Number } \\
\text { of active } \\
\text { planners }\end{array}$} & \multicolumn{2}{|c|}{ Percentage of active planners $(95 \% \mathrm{Cl})$} & \multirow[b]{2}{*}{ Unadjusted OR (95\% Cl) } & \multirow[b]{2}{*}{ Adjusted OR ${ }^{\mathrm{a}}(95 \% \mathrm{Cl})$} \\
\hline & & $\begin{array}{l}\text { Stopped } \\
\text { contraception }<1 \text { year } \\
\text { ago } \\
(n=42,961)\end{array}$ & $\begin{array}{l}\text { Stopped } \\
\text { contraception }>1 \text { year } \\
\text { ago } \\
(n=39,912)\end{array}$ & & \\
\hline \multicolumn{6}{|l|}{ Age $(n=81,533)$} \\
\hline $18-24$ & 25,038 & $33.2 \%(32.7-33.6)$ & $28.0 \%(27.6-28.5)$ & 1.00 (ref) & 1.00 (ref) \\
\hline $25-34$ & 44,732 & $55.9 \%(55.4-56.4)$ & $53.8 \%(53.3-54.3)$ & $1.14(1.10-1.18)$ & $1.27(1.22-1.32)$ \\
\hline $35-40$ & 10,456 & $9.9 \%(9.6-10.2)$ & $16.0 \%(15.6-16.4)$ & $1.91(1.83-2.00)$ & $2.17(2.05-2.30)$ \\
\hline $41+$ & 1307 & $1.0 \%(0.9-1.1)$ & $2.2 \%(2.1-2.4)$ & $2.53(2.25-2.85)$ & $2.88(2.52-3.29)$ \\
\hline Question not answered & 1340 & - & - & - & - \\
\hline \multicolumn{6}{|l|}{ BMI $(n=71,373)$} \\
\hline 18.5-24.9 (Recommended) & 28,662 & $42.4 \%(41.9-42.9)$ & $37.7 \%(37.2-38.3)$ & 1.00 (ref) & 1.00 (ref) \\
\hline$<18.5$ (Underweight) & 2304 & $3.1 \%(2.9-3.3)$ & $3.4 \%(3.2-3.5)$ & $1.21(1.11-1.32)$ & $1.19(1.09-1.29)$ \\
\hline 25-29 (Overweight) & 18,443 & $26.6 \%(26.1-27.0)$ & $25.0 \%(24.6-25.5)$ & $1.06(1.02-1.10)$ & $1.04(1.00-1.08)$ \\
\hline $30+($ Obese $)$ & 21,964 & $27.9(27.5-28.4)$ & $33.9 \%(33.4-34.4)$ & $1.36(1.32-1.41)$ & $1.30(1.24-1.36)$ \\
\hline $\begin{array}{l}\text { Question not answered/BMl } \\
\text { excluded }\end{array}$ & 11,500 & - & - & - & - \\
\hline \multicolumn{6}{|l|}{ Smoking $(n=81,737)$} \\
\hline No & 64,014 & $80.3 \%(79.9-80.6)$ & $76.2 \%(75.8-76.6)$ & 1.00 (ref) & 1.00 (ref) \\
\hline Yes & 17,723 & $19.7 \%(19.4-20.1)$ & $23.8 \%(23.4-24.2)$ & $1.27(1.23-1.31)$ & $1.25(1.20-1.31)$ \\
\hline Question not answered & 1136 & - & - & - & - \\
\hline \multicolumn{6}{|c|}{ Illicit or recreational drug use $(n=81,678)$} \\
\hline No & 78,813 & $96.8 \%(96.6-96.9)$ & $96.2 \%(96.0-96.4)$ & 1.00 (ref) & 1.00 (ref) \\
\hline Yes & 2865 & $3.2 \%(3.1-3.4)$ & $3.8 \%(3.6-4.0)$ & $1.18(1.09-1.27)$ & $1.17(1.07-1.28)$ \\
\hline Question not answered & 1195 & - & - & - & - \\
\hline \multicolumn{6}{|c|}{ Alcohol consumption $(n=81,659)$} \\
\hline No & 41,532 & $47.5 \%(47.1-48.0)$ & $54.4 \%(53.9-54.9)$ & 1.00 (ref) & 1.00 (ref) \\
\hline Yes & 40,127 & $52.5 \%(52.0-52.9)$ & $45.6 \%(45.1-46.1)$ & $0.76(0.74-0.78)$ & $0.74(0.72-0.77)$ \\
\hline Question not answered & 1214 & - & - & - & - \\
\hline \multicolumn{6}{|c|}{ Caffeine consumption $(n=81,568)$} \\
\hline No & 17,278 & $20.4 \%(20.0-20.8)$ & $22.0 \%(21.6-22.4)$ & 1.00 (ref) & 1.00 (ref) \\
\hline Yes & 64,290 & $79.6 \%(79.2-80.0)$ & $78.0 \%(77.6-78.4)$ & $0.91(0.88-0.94)$ & $0.94(0.90-0.98)$ \\
\hline Question not answered & 1305 & - & - & - & - \\
\hline \multicolumn{6}{|c|}{ Weekly level of activity $(n=75,076)$} \\
\hline Less than 150 min of activity & 32,002 & $42.9 \%(42.4-43.3)$ & $42.4 \%(41.9-42.9)$ & 1.00 (ref) & 1.00 (ref) \\
\hline $\begin{array}{l}\text { At least } 150 \text { min of moder- } \\
\text { ate activity }\end{array}$ & 32,291 & $43.1 \%(42.6-43.6)$ & $43.0 \%(42.4-43.5)$ & $1.01(0.98-1.04)$ & $1.03(1.00-1.07)$ \\
\hline $\begin{array}{l}150 \text { or more minutes of } \\
\text { vigorous activity }\end{array}$ & 10,783 & $14.1 \%(13.7-14.4)$ & $14.7 \%(14.3-15.0)$ & $1.05(1.01-1.10)$ & $1.08(1.02-1.13)$ \\
\hline Question not answered & 7797 & - & - & - & - \\
\hline \multicolumn{6}{|c|}{ Daily consumption of five portions of fruit and vegetables $(n=74,403)$} \\
\hline No & 23,144 & $30.7 \%(30.2-31.1)$ & $31.6 \%(31.1-32.1)$ & 1.00 (ref) & 1.00 (ref) \\
\hline Yes & 40,216 & $54.8 \%(54.3-55.3)$ & $53.2 \%(32.7-53.7)$ & $0.94(0.91-0.97)$ & $0.93(0.90-0.97)$ \\
\hline Don't know & 11,043 & $14.5 \%(14.2-14.9)$ & $15.2 \%(14.8-15.6)$ & $1.02(0.97-1.06)$ & $1.03(0.98-1.09)$ \\
\hline Question not answered & 8470 & - & - & - & - \\
\hline \multicolumn{6}{|c|}{ Folic acid supplementation $(n=63,541)$} \\
\hline No & 35,561 & $54.9 \%(54.4-55.5)$ & $57.1 \%(56.5-57.7)$ & 1.00 (ref) & 1.00 (ref) \\
\hline Yes & 27,980 & $45.1 \%(44.5-45.6)$ & $42.9 \%(42.3-43.5)$ & $0.92(0.89-0.95)$ & $0.88(0.85-0.91)$ \\
\hline Question not answered & 19,332 & - & - & - & - \\
\hline
\end{tabular}

a models adjusted for age, BMI, smoking, drug use, alcohol consumption, caffeine consumption, activity level, consumption of five-a-day \& folic acid supplementation 
around 2000 pregnancies affected by neural tube defects between 1998 and 2012 would have been prevented if fortification had been in place [23]. In June 2019, the UK government issued a public consultation on mandatory fortification with a set of questions on its effectiveness and feasibility [24], the outcome of which is awaited with interest.

Amongst the respondents, nearly $50 \%$ were overweight or obese which is similar to UK women of reproductive age. Obesity affects oocyte function, early embryonic development, fetal growth and complications in pregnancy and at delivery [4]. In addition, sub-optimal nutrition before pregnancy may adversely impact pregnancy outcomes by increasing the risk of micronutrient deficiencies implicated in fetal growth and development [1]. Furthermore, inadequate physical activity before pregnancy may increase the risk of gestational diabetes and pre-eclampsia [25].

Given that many pre-existing health conditions, as well as health problems in a previous pregnancy increase the risk of adverse pregnancy outcome, it was notable that of the $15 \%$ of women declaring ongoing health conditions or health issues in a previous pregnancy, only half had sought clinical advice before considering pregnancy.

It is of little surprise that increasing age, and underweight and obese BMI were associated with reduced fertility, further emphasising the need for targeted health messaging and intervention.

High-risk behaviours were common in younger women; this was illustrated by a high prevalence of smoking and drug use as well as a lack of folic acid supplementation in the 18 to 24 age group actively planning pregnancy, and aligns with a US study $(n=847)$ which observed a high prevalence of long term binge drinking and smoking in this age group [26]. A systematic review also reported that younger women were less aware of the importance of folic acid supplementation [27], and as evidenced by analysis of hair samples, younger women are more likely to use recreational drugs in the periconceptual period [28]. The Preconception Partnership's proposal for preconception health highlighted the presence of social and medical risks in younger women and a need for more effective methods to engage and support this group [2].

\section{Strengths and limitations}

Our study has a number of strengths. It is the largest sample of health behaviours and lifestyle factors in women planning a pregnancy studied to date, and the breadth of questions provides a wealth of information. A high proportion of the women who used the digital preconception tool completed the questionnaire, perhaps reflecting the user friendliness of the online platform. Studies with a self-selected sample such as this, are recognised to be subject to social desirability bias where high-risk health behaviours may be underreported [7]. However, the high prevalence of these behaviours, and the wide geographical distribution suggests that the tool was successful in targeting harder to reach groups.

We acknowledge several limitations. The brevity of the questionnaire, whilst likely to be instrumental in the wide response, inevitably restricted the scope of analysis. Information on socio-economic status and ethnicity was lacking. When reporting health behaviours such as drinking alcohol, women were not asked the frequency of consumption which would have enriched data interpretation. There may also be an element of volunteer bias to the responses, as those using the digital tool were likely to include a subgroup actively seeking advice on their preconception health behaviours, and therefore may not be representative of all UK women planning a pregnancy. Anonymised IP addresses are associated with some inaccuracy when identifying location. Lastly, an assumption was made that women utilising the tool were planning a pregnancy.

\section{Conclusion}

This unique study presents health behaviours of the largest group of women planning a pregnancy to date, including a novel insight into behaviours amongst women trying to conceive for less than, and over one year. We have clearly shown that women are not adequately preparing for pregnancy, evidenced by a high prevalence of smoking, low folic acid use, low levels of physical activity and inadequate fruit and vegetable intake. Smoking and lack of folic acid were particularly common in both younger women and underweight women and in those trying to conceive for over a year. Underlying medical conditions were common yet clinical advice rarely sought. This study highlights the importance of targeted support for such women planning pregnancy. Data from this study will contribute metrics for public health planning as suggested by the Preconception Partnership [2].

\section{Abbreviations \\ PHE: Public Health England; RCOG: Royal College of Obstetricians and Gynaecologists.}

\section{Acknowledgements \\ We gratefully acknowledge the contribution of all women planning preg- nancy who completed the questionnaire.}

Authors' contributions

SLW and ACF conceptualised and designed the study. BM and KK analysed the data. BM wrote the first draft of the manuscript with substantial input from 
SLW, ACF, KK, JS and LP. All authors saw successive drafts of the paper and provided input. All authors approved the final version of the manuscript.

\section{Funding}

The Tommy's Planning for Pregnancy tool and accompanying social media campaign was funded by PHE, Tommy's and RCOG. Tommy's also provide salary support for ACF, SLW and LP. Neither PHE, Tommy's nor RCOG had any role in data analysis or interpretation, nor in the writing of the report or the decision to submit the paper for publication.

\section{Availability of data and materials}

The datasets used and/or analysed during the current study are available from the corresponding author on reasonable request.

\section{Declarations}

\section{Ethics approval and consent to participate}

Individual participant data that underlie the results reported in this article, after de-identification will be available on request, beginning 9 months and ending 36 months following article publication, to investigators whose proposed use of the data has been approved by an independent review committee (KCL Women's Health Review Committee).

\section{Consent for publication}

$$
\text { Not applicable. }
$$

\section{Competing interests}

The authors declare no competing interests.

\section{Author details}

${ }^{1}$ Department of Mathematics and Statistics, University of Strathclyde, Glasgow, UK. ' ${ }^{2}$ GA Institute for Women's Health, University College London, 74 Huntley Street, WC1E 6AU London, UK. ${ }^{3}$ Department of Women and Children's Health, School of Life Course Sciences, King's College London, 10th Floor North Wing, St Thomas'Hospital, Westminster Bridge Road, SE1 7EH London, UK. ${ }^{4}$ Department of Nutritional Sciences, Franklin-Wilkins Building, 150 Stamford Street, London SE1 9NH, UK.

\section{Received: 11 March 2021 Accepted: 13 July 2021}

Published online: 28 July 2021

\section{References}

1. Stephenson J, Heslehurst N, Hall J, et al. Before the beginning: nutrition and lifestyle in the preconception period and its importance for future health. Lancet. 2018;391:1830-41.

2. Stephenson J, Vogel C, Hall J, et al. Preconception health in England: a proposal for annual reporting with core metrics. Lancet. 2019;393:2262-71.

3. Chivers B, Boyle J, Lang A, Teede H, Moran L, Harrison C. Preconception health and lifestyle behaviours of women planning a pregnancy: a crosssectional study. J Clin Med. 2020;9:1701.

4. Public Health England. Making the case for preconception care. London: PHE Publications; 2018.

5. Goodfellow A, Frank J, McAteer J, Rankin J. Improving preconception health and care: a situation analysis. BMC Health Serv Res. 2017;17(1):595. https://doi.org/10.1186/s12913-017-2544-1.

6. Ojukwu O, Patel D, Stephenson J, Howden B, Shawe J. General practitioners' knowledge, attitudes and views of providing preconception care: a qualitative investigation. Ups J Med Sci. 2016;121:256-63.

7. Stephenson J, Patel D, Barrett G, et al. How do women prepare for pregnancy? Preconception experiences of women attending antenatal services and views of health professionals. PLoS ONE. 2014;9:e103085.

8. Earle S, Tariq A, Komaromy C, et al. Preconception care for women with type 1 or type 2 diabetes mellitus: a mixed-methods study exploring uptake of preconception care. Health Technol Assess Rep. 2017;21:1-130.

9. van Elten T, Karsten M, Geelen A, et al. Preconception lifestyle intervention reduces long term energy intake in women with obesity and infertility: a randomised controlled trial. Int J Behav Nutr Phys Act. 2019;16. https://doi.org/10.1186/s12966-018-0761-6.

10. Hussein N, Qureshi N, Kai J. The effects of preconception interventions on improving reproductive health and pregnancy outcomes in primary care: a systematic review. Contraception. 2014;90:339.

11. Skogsdal Y, Fadl H, Cao Y, Karlsson J, Tydén T. An intervention in contraceptive counselling increased the knowledge about fertility and awareness of preconception health-a randomized controlled trial. Up J Med Sci. 2019;124:203-12.

12. Hemsing N, Greaves L, Poole N. Preconception health care interventions: a scoping review. Sex Reprod Healthc. 2017:14:24-32.

13. Body mass index - BMI. Euro.who.int. 2020. https://www.euro.who.int/ en/health-topics/disease-prevention/nutrition/a-healthy-lifestyle/bodymass-index-bmi. Accessed 2 Aug 2020.

14. National Diet and Nutrition Survey. GOV.UK. 2016. https://www.gov.uk/ government/collections/national-diet-and-nutrition-survey. Accessed 14 Nov 2020.

15. Roser M, Appel C, Ritchie H. Human height. Our world in data. 2019 https://ourworldindata.org/human-height. Accessed 22 Nov 2019.

16. CDC Weight for Age Percentiles for Girls (2-20 years). Reference.medscape.com. 2019. https://reference.medscape.com/calculator/659/cdcweight-for-age-percentiles-for-girls-2-20-years. Accessed 22 Nov 2019.

17. Poston $L$, Bell $R$, Croker $H$, et al. Effect of a behavioural intervention in obese pregnant women (the UPBEAT study): a multicentre, randomised controlled trial. Lancet Diabetes Endocrinol. 2015;3:767-77.

18. Brough L, Rees G, Crawford M, Dorman E. Social and ethnic differences in folic acid use preconception and during early pregnancy in the UK: effect on maternal folate status. J Hum Nutr Diet. 2009;22:100-7.

19. Births in England and Wales: summary tables - Office for National Statistics. 2020. https://www.ons.gov.uk/peoplepopulationandcommun ity/birthsdeathsandmarriages/livebirths/datasets/birthsummarytables. Accessed 15 Nov 2020

20. Wellings $\mathrm{K}$, Jones $\mathrm{KG}$, Mercer $\mathrm{CH}$, et al. The prevalence of unplanned pregnancy and associated factors in Britain: findings from the third National Survey of Sexual Attitudes and Lifestyles (Natsal-3). Lancet. 2013;382:1807-16.

21. Adult smoking habits in England - Office for National Statistics. 2020. https://www.ons.gov.uk/peoplepopulationandcommunity/healthands ocialcare/healthandlifeexpectancies/datasets/adultsmokinghabitsin england. Accessed 15 Nov 2020.

22. Inskip H, Crozier S, Godfrey K, Borland S, Cooper C, Robinson S. Women's compliance with nutrition and lifestyle recommendations before pregnancy: general population cohort study. BMJ. 2009;338:b481-b481.

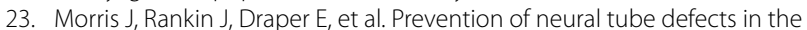
UK: a missed opportunity. Arch Dis Childh. 2015;101:604-7.

24. Wald N, Morris J, Blakemore C. Urgent need for folic acid fortification of flour and grains: response to the 2019 UK Government's public consultation. Arch Dis Childh. 2019:105:6-9.

25. Hegaard H, Pedersen B, Bruun Nielsen B, Damm P. Leisure time physical activity during pregnancy and impact on gestational diabetes mellitus, pre-eclampsia, preterm delivery and birth weight: a review. Acta Obstet Gynecol Scand. 2007;86:1290-6.

26. Chuang C, Weisman C, Hillemeier M, Schwarz E, Camacho F, Dyer A. Pregnancy intention and health behaviors: results from the Central Pennsylvania Women's Health Study Cohort. Matern Child Health J. 2009:14:501-10.

27. Stockley L, Lund V. Use of folic acid supplements, particularly by lowincome and young women: a series of systematic reviews to inform public health policy in the UK. Public Health Nutr. 2008;11:807-21.

28. David A, Holloway A, Thomasson L, et al. A case-control study of maternal periconceptual and pregnancy recreational drug use and fetal malformation using hair analysis. PLOS ONE. 2014;9:e111038.

\section{Publisher's Note}

Springer Nature remains neutral with regard to jurisdictional claims in published maps and institutional affiliations. 